\title{
Computer Vision-Based Gait Velocity from Non-Obtrusive Thermal Vision Sensors
}

\author{
$1^{\text {st }}$ Javier Medina-Quero \\ Department of Computer Science \\ University of Jaén \\ Jaén, Spain \\ jmquero@ujaen.es \\ $4^{\text {th }}$ Joseph Rafferty \\ Connected Health Innovation Centre \\ Ulster University \\ Jordanstown, U.K. \\ j.rafferty@ulster.ac.uk
}

\author{
$2^{\text {nd }}$ Colin Shewell \\ Connected Health Innovation Centre \\ Ulster University \\ Jordanstown, U.K. \\ cp.shewell@ulster.ac.uk \\ $5^{\text {th }}$ Chris Nugent \\ Connected Health Innovation Centre \\ Ulster University \\ Jordanstown, U.K. \\ cd.nugent@ulster.ac.uk
}

\author{
$3^{\text {rd }}$ Ian Cleland \\ Connected Health Innovation Centre \\ Ulster University \\ Jordanstown, U.K. \\ i.cleland@ulster.ac.uk
}

\author{
$6^{\text {th }}$ Macarena Espinilla Estévez \\ Department of Computer Science \\ University of Jaén \\ Jaén, Spain \\ mestevez@ujaen.es
}

\begin{abstract}
Gait velocity is an important measure of independence and functional ability to those within the older population. Detecting changes in gait velocity can aid to provide interventions to avoid hospitalisation, currently gait velocity is assessed in a clinical setting, where the patient is timed over a measured distance between 3-6 metres by a clinician, however, this is time consuming, subjective, and not possible to carry out frequently over time. An unobtrusive method of monitoring gait velocity, frequently, over extended periods of time, would therefore be advantageous when developing interventions. This paper proposes an unobtrusive computer vision-based method of continuously monitoring an occupants gait velocity within their own home. This is achieved through the use of a low cost thermal vision sensor. The system was benchmarked against the clinical standard method of being timed by a stopwatch. Results show a high correlation between the gait velocity measured by the thermal vision sensor and the measured stopwatch velocity $(\mathbf{R}=\mathbf{0 . 9 4 1}, \mathbf{p}=\mathbf{0 . 0 2})$.
\end{abstract}

Index Terms-Thermal vision sensors, Ambient assisted living, Smart homes, Gait recognition

\section{INTRODUCTION}

Mobility is a key aspect of keeping independence and functional ability within the elderly population [1], as well as being a general indicator of their health [2], [3]. Gait velocity in particular can aid to predict multiple conditions that may lead to hospitalisation, such as chronic obtrusive pulmonary disease, congestive heart failure [4], [5], fall risk likelihood [6], and even a predictor of the onset of cognitive decline [1].

The traditional approach to measure gait velocity involves the patient walking over a set distance while being timed by a clinician [7]; however, there are limitations to this method. Walking in a clinic based environment while under observation may not be an accurate representation of how the patient would walk in a general day-to-day manner at home [8]. There is

This research has received funding under the REMIND project Marie Sklodowska-Curie EU Framework for Research and Innovation Horizon 2020 , under Grant Agreement No. 734355 The authors would also like to thank the Connected Health Innovation Centre (CHIC). also the issue that these clinic tests will only be administered at a time were the patient is available to attend the clinic. At the most frequent this would still only dictate that the measurement is carried out at a frequency of three to six months. This scenario does not provide enough granularity to monitor trends in their gait velocity in order to provide any responsive intervention, nor does it offer opportunity to rapidly change a patients medication/treatments in response to how it is effecting their mobility.

Multiple attempts have been made to design a system that will allow gait analysis to be performed within the occupant's own home on a continuous basis [10]. Many of these systems require the occupant to carry or wear a device at all times. Doing so, they can be unreliable as older adults may feel uncomfortable and encumbered by wearable devices [9], [11]. This can result in the devices being used either incorrectly or not at all. This is further compounded by the fact that off-the-shelf wearable devices, such as FitBit, are not able to capture gait velocity. Other solutions, such as smartphone enabled GPS, are not accurate enough to work indoors [12]. Camera systems mounted in the occupant?s home have also been considered, however, traditional cameras often have a limited field of view, suffer from occlusions, and raise privacy concerns from the occupant and their families perspective.

This paper proposes a method of computing the gait velocity of an inhabitant through the use of a non-obtrusive device: a Thermal Vision Sensor (TSV). The proposed method does not require that the inhabitant to carry a wearable sensor or device. The TSV used in this approach is a Heimann HTPA $32 \times 31$ [13], its low resolution offers a degree of privacy not offered by traditional camera methods as occupants cannot be identified providing a more positive acceptance than standard vision cameras [14].

The remainder of the paper is structured as follows: in Section II, previous related works are presented; in Section III, we detail a computer vision-based methodology to compute the 
gait velocity of an inhabitant using a TSV; in Section IV, a case study and related evaluation and results are presented; finally, in Section V, conclusions and future work are proposed.

\section{RELATED WORK}

This Section presents a summary of the current state-of-theart of gait analysis within the home. A number of studies are reviewed, which utilise diverse approaches based on sensor technologies to obtain a gait analysis. While the findings are promising, there exists a number of challenges which hope to be addressed within the proposed work.

Hayes et al. [1] propose the use of passive infrared sensors (PIR) within an occupant's home to measure gait velocity on a regular basis. They evaluated their system against a GAITRite gait mat using a timed walk test with 18 subjects, they found the velocity measured by their system was highly correlated to the measured walking velocity in all cases, using two sensors they achieved $\mathrm{R}=0.93$, three sensors $\mathrm{R}=0.96$, four sensors $\mathrm{R}=0.97$, and five sensors $\mathrm{R}=0.99$. While these results are encouraging there are limitations encountered with this system. The main one being the number of sensors that are required in order to get an accurate reading, with six sensors in total being used, thus driving up cost and complexity of install and maintenance, which requires an expensive calibration each time it is installed in a new location.

Hsu et al. [15] developed a system to measure gait velocity within the home using radio signals to continuously measure gait velocity. Their WiGait system uses a sensor which is placed on a wall within the room and does not require any device to be worn on the occupant's person. They compared their system against the VICON system using 18 subjects, the average error rates of $1.9 \%$ and $4.2 \%$ for gait velocity and stride length respectively. A further study was carried out with 25 participants, they were asked to walk along a seven metre line with their gait velocity being measured over the central five metre section, allowing one metre at the start and end for acceleration/deceleration time. WiGait's accuracy in determining gait velocity varied between $85.9 \%$ and $99.8 \%$. However, their system requires a large amount of effort in installing and any movement in the occupants limbs is measured as a movement of the whole body thus it can give false readings of movement/velocity.

McGinnis et al. [16] proposed a system to estimate gait velocity using an array of skin mounted, conformal accelerometers. They tested this system on both subjects with normal gait and those with gait impairments induced by multiple sclerosis. For normal gait the system overestimates by 0.01 $\mathrm{m} / \mathrm{s}$ and achieves a root mean square error of $0.12 \mathrm{~m} / \mathrm{s}$. Results of a Bland-Altman analysis showed results within a $95 \%$ confidence interval. The main limitation of this system is the requirement of the occupant to wear multiple sensors on their person, particularly as members of the older population do not feel comfortable wearing wearable devices [9], [11], this is further compounded in this case as the sensors have to be worn next to the skin thus increasing the discomfort.
The proposed system in this paper attempts to avoid the limitations found in this Section. Through the use of a single low cost, easy to install ceiling mounted TSV aims to: i) achieve performance on par with the reviewed systems, ii) remove the requirement of the inhabitant to have to wear any wearable device in order for the system to function. Furthermore, the low resolution from the TSV provides a degree of privacy which is not offered by traditional camera based systems, while still providing the accuracy for computer vision processing in order to perform gait analysis.

\section{Methodology}

As discussed in Section I, this paper presents an computer vision-based gait velocity estimation of inhabitants using a TSV. In order to provide a clear field of view in the frame, the TSV is located in the room roof collecting an aerial view of the occupants, this also offers the advantage of reducing occlusions. For each frame, the data collected by TVS are defined by a $32 \times 31$ matrix, where each pixel value is in the range between 0 and 255 .

The main steps for generating the computer vision-based gait velocity are:

- Analysing distortion and calibrating the TVS in order to calculate the affine transformation, which relates the pixel coordinates from the TSV to the real world.

- Processing of the thermal frames to detect the central location of inhabitants blob in the scene.

- Real-time tracking of the location of an occupant while they are walking in the scene from the sequence of frames collected by TVS.

These stages are further detailed in the following section.

\section{A. Distortion and Affine Transformation}

To evaluate the potential distortion from TVS, an initial calibration procedure was carried out. A symmetrical grid of points were mapped out as references in the real world location, these have been measured by TVS for further evaluation.

The grid of points has been deployed by thermal markers on the floor of the scene under the TVS. They were located in a symmetrical grid of points separated by 0.5 metres, as shown in Figure 1. It should be noted that avoiding the angle rotation, between field of view from TSV and the symmetrical grid, helps in evaluating the distortion and the affine transformation to real world.

Firstly, in order to evaluate the potential distortion, the coordinates of measured points in the image from TVS were related the coordinates in the real world location of the grid points. A constant linear progression was obtained (see Figure 1) from measured coordinates in the centre, where the TVS is located in a aerial view, to the measured coordinates in peripheral points within the symmetrical grid of points. The results from this showed an effective factory calibration, with no distortion by the fish-eye lens.

Secondly, in order to calculate the affine transformation from the TVS to real world using homogeneous coordinates, the following equation is used: 
A)

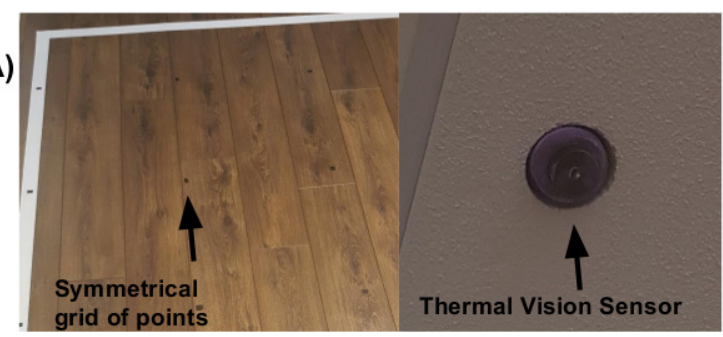

Relation in $\mathrm{X}$ axis between real-world location of grid points and affine transformation of grid-coordinate pixels

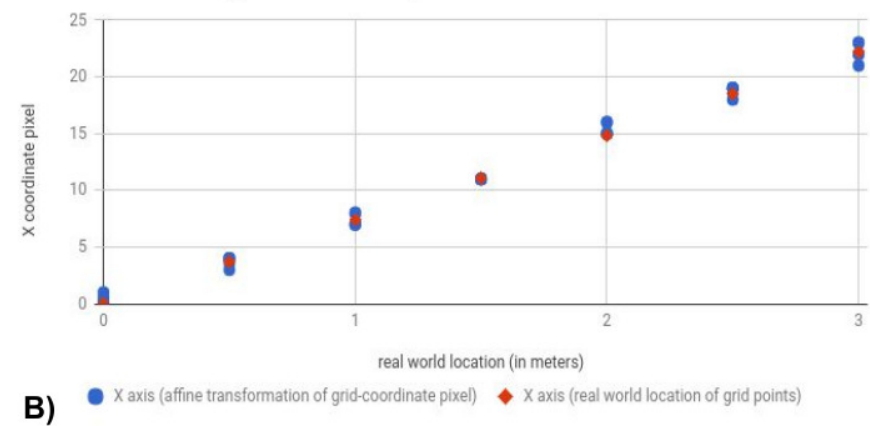

Fig. 1. A) Symmetrical grid of points in the floor and TSV cenital location in roof. B) Relation in $X$ axis between real-world location of grid points and affine transformation of grid-coordinate pixels

$$
\left(\begin{array}{l}
x^{*} \\
y^{*}
\end{array}\right)=H \times\left(\begin{array}{l}
x \\
y
\end{array}\right)+B
$$

where $x^{*}, y^{*}$ are the real-world coordinates, $x, y$ the TSV coordinates, $H$ a matrix transformation and $B$ and offset vector. In the case of the implemented TVS, the affine transformation is computed using a linear regression which translates the thermal coordinates from the pixel in the frame of the TVS in regard to the real world location. It has been defined by a straightforward scale operation due to: i) avoiding rotation between TVS and grid of points in the real world, and ii) the centred and aerial view from the roof, obtaining a pure scale transformation:

$$
\left(\begin{array}{l}
x^{*} \\
y^{*}
\end{array}\right)=\left[\begin{array}{cc}
S_{x} & 0 \\
0 & S_{y}
\end{array}\right] \times\left(\begin{array}{l}
x \\
y
\end{array}\right), S_{x}=S_{y}=0.1352
$$

The relation in the $X$ axis between the straightforward transformation of coordinate pixels from the TVS and the real world location, is show in Figure 1.

\section{B. Detecting Central Location of an Inhabitant}

In order to detect the location of an inhabitant from the aerial view of the TVS, a real-time processing of frames based on computer vision has been developed. The computer visionbased process includes next consecutive steps to compute each single frame:

- Thresholding binarization [17]. A segmentation of frames based on a minimal threshold $\min _{v}$ is included. The noise values, which are lower than the threshold and not related to an inhabitant detection are set to zero; in another case,

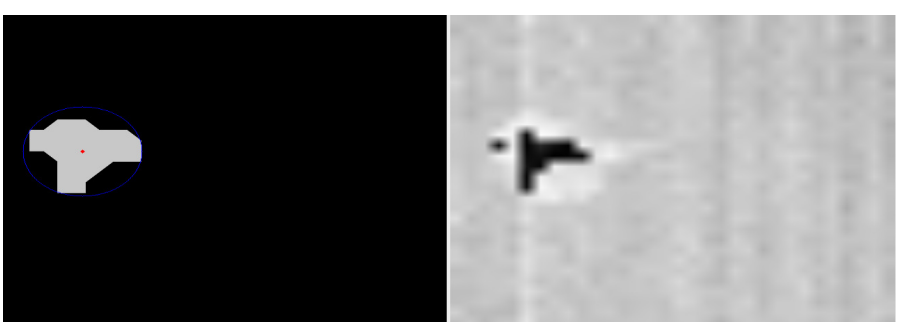

Fig. 2. Right) Original frame from TVS. Left) Minimal enclosing circle as output from the computer vision process (ratio in blue and centre).

they are set to 1 . An empirical value of $\min _{v}=50$ was defined.

- Gaussian blur. A smoothing filtering to reduce the saltand-pepper noise is included.

- The well-known algorithm to find contours [18] is applied to detect the presence of an inhabitant. A minimal size of the object $2 x 2$ is required to filter small or isolated objects, such as lamps, hands or feet.

- Due to the shape of the aerial view of inhabitants, a minimal circle is proposed to enclose the points of the contour detected in previous step.

This minimal enclosing circle (MEC) represents the output of the single-frame processing. In figure 2 , an example of original frame and MEC is shown.

\section{Real-Time Tracking of the Inhabitant Location}

For each image collected by the TSV, computer vision tracking was developed to trace the inhabitant between frames in real-time in order to generate the tracking history and compute the gait velocity. This tracking enables the collection of the distance, time, and path of the inhabitant while they are walking in the scene.

A lightweight real-time tracking for a single inhabitant is proposed, as the aim is analysing the gait velocity in a controlled context. This algorithm tracks the inhabitant when he/she appears in the scene and it shows the walking metrics of the inhabitant when he/she disappears from the scene.

For that, the proposed tracking collects and processes in real-time the frames from the TVS applying the process described in Section III-B in a first step. Secondly, while the inhabitant is walking in the scene, the real-time tracking obtains the central location of the inhabitant and the timestamp of the frame, which are recorded in the tracking history. At the end, this is when the inhabitant is not detected in the current frame, the time and straight distance is computed (as the difference between entry point and the exit point, and first and last time respectively). Both are the key metrics for analysing the gait velocity in Section IV. Finally, the Kalman filter [19] is applied to the path in order to compute an smooth approximation of the walking path of the inhabitant. Algorithm 1, details the pseudo-code of the real-time tracking of the occupant location.

Figure 3, presents an example of real-time tracking collected by the TVS. 
Data: newFrame, history

Result: updated history or metrics

object=MEC(newFrame);

if object $=\emptyset$ then

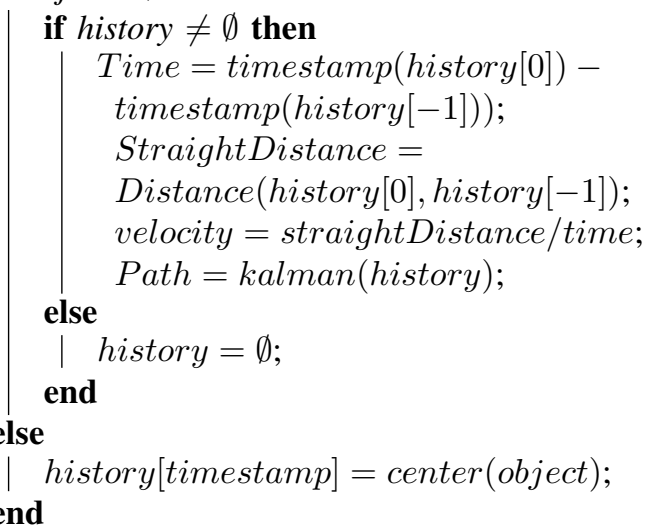

Algorithm 1: Real-time tracking algorithm.

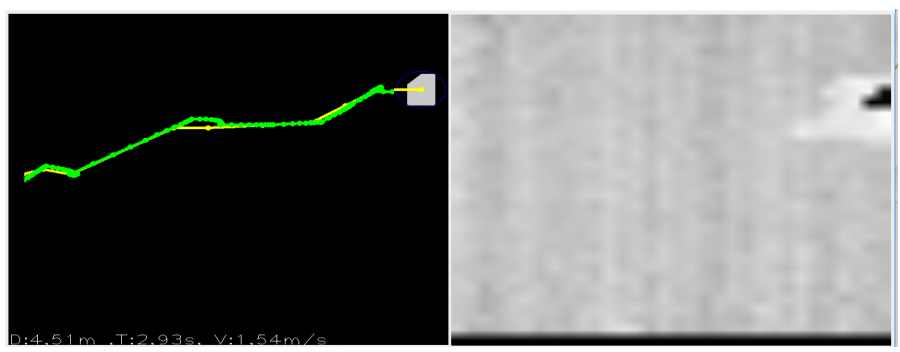

Fig. 3. Left) In yellow and green lines, the path from tracking history and Kalman filtered path are plotted respectively. The distance, time, and velocity are detailed as output in the bottom of the figure. Right) Original frame from TVS at the end of the path.

\section{Experimental Setup And Results}

This section describes a case study where 42 tests of gait velocity where carried out by seven participants. The aim of the study was to compare the gait velocity metrics obtained for the approach in regard to an human observer with a stopwatch.

For that, a straight line of 3.1 metres was mapped out on the floor within the field of view area of the TVS installed in an aerial view. In each case, the participants walked across the straight line at different velocities (low, normal, and fast). A human observer collected the time they spent walking using a stopwatch, which provides the velocity as a quotient of the length of the line (3.1 metres) and the collected time.

The case study was carried out by seven participants (one female, six male). The averages and standard deviations of the height and age of the participants are $\mu=175.57 \mathrm{~cm}$, $\sigma=10.60 \mathrm{~cm}$ and $\mu=31.6 y, \sigma=6.2 y$, respectively. In order to provide a range of situations regardless of the age and vitality of the participants, they walked at slow, normal and, fast velocity along the straight line. Each velocity was repeated twice resulting in 42 independent gait velocity tests.

The stopwatch collected velocity has been compared with the TSV based velocity obtained using the methodology described in Section III. We note that the distance and time stopwatch speed vs thermal sensor speed

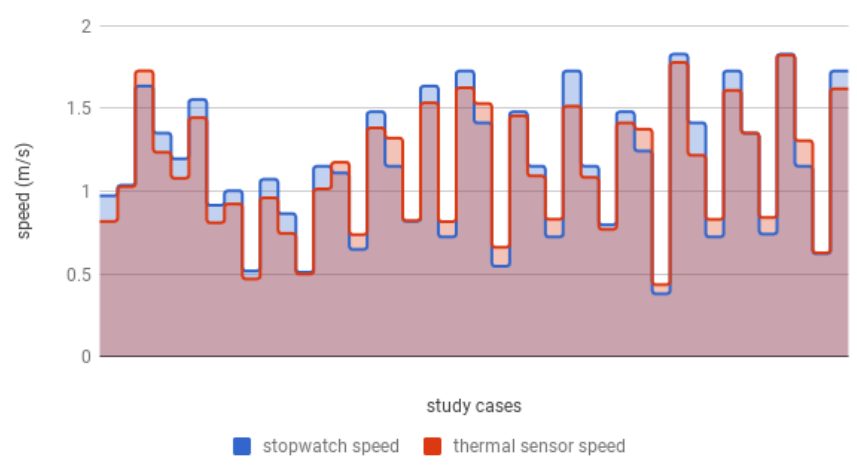

Fig. 4. Comparison between the stopwatch-collected velocity and the TSVbased velocity in the 42 cases.

TABLE I

MAE AND RMSE FROM CASE STUDY.

\begin{tabular}{|l|l|}
\hline Error & Value \\
\hline$M A E$ & $\mathbf{0 . 0 8 9 7} \mathrm{m}$ \\
\hline$R M S E$ & $\mathbf{0 . 1 0 3 1} \mathrm{m}^{2}$ \\
\hline
\end{tabular}

collected by the TSV do not require to be similar to the stopwatch because of the reference line is only partially included within the vision area of the TSV. Due to this, the real-time tracking, from the first-entrance to the last-exit points of the inhabitant, could provide a higher distance and a longer time than stopwatch but keeping the constant relation of velocity closely related.

\section{A. Evaluation}

Figure 4 presents a visual comparison between the stopwatch velocity and the TSV based velocity in the 42 cases.

For a further evaluation, a statistical analysis was performed using SPSS (IBM, SPSS Statistics, Version 22). Statistical significance was set to $p<0.05$. Initially a one sample Ttest was computed to detect statistical significant differences in gait velocity measured by the human observer (stopwatch) and the TVS. Bland-Altman [20] plots were constructed to examine the level agreement between the two measures. The Bland-Altman limits of agreement (mean \pm 1.96SD) for the difference between measurement techniques which provides an indication of the minimal detectable difference in the estimated velocity. To further assess the level of agreement and to identify any underlying bias in the measurement, linear regression analysis was undertaken. The intercept (b) indicates the systematic difference between measurement techniques, and the slope $(\mathrm{m})$ indicates how closely one measurement technique is able to detect changes in the other.

\section{B. Results}

The Mean Absolute Error (MAE) and Root Mean Square Error (RMSE) from the 42 cases are shown in Table I, which indicates a good performance of the approach. 
One sample T-test showed no statistically significant difference between the two velocity measurements $(p=0.05)$. The Bland-Altman and regression plots 5, highlight the unbiased velocity estimate provide by the TVS. Each point of the BlandAltman plot represents a single walking velocity test, where the $y$-coordinate is the difference in the velocity estimate between the two methods and the $\mathrm{x}$-coordinate is the average of the two methods. The dashed red line indicates the mean difference in the velocity estimate, and the dashed black lines indicate the limits of agreement. The difference in gait velocity detected by the human observer and the TVS all lie within the limits of agreement. This signifies good stability of the gait velocity detection from the TVS across all velocities. Similarly, each point of the regression plot represents a single walking velocity test, where the y-coordinate is the estimated velocity and the $\mathrm{x}$-coordinate is the true velocity. The line of best fit is plotted as a dashed red line. This shows a high correlation between the 2 measurements $(\mathrm{R}=0.941, \mathrm{p}=0.02)$.

\section{CONCLUSIONS}

In this work, a real-time gait velocity estimation based on computer vision from a TSV is detailed. The main key points of the approach are: i) using a low-cost TSV which guarantees the user privacy, ii) a aerial location for a straightforward affine calibration, iii) a single frame processing based on computer vision, iv) a real-time tracking for analysing gait velocity metrics.

A study with 42 cases shows the encouraging performance for analysing the gait velocity in regard to an human observer with a stopwatch. The one sample T-test showed no statistically significant difference between the two velocity measurements.

Future works will be focused on including real-time tracking with several TSV with overlapping areas of vision, as well as, designing and evaluating a real-time multiple-occupancy tracker. Moreover, the detection of fall by means of Convolutional Neural Networks will be analysed.

\section{REFERENCES}

[1] Hayes, T. L., \& Hagler, S., \& Austin, D., \& Kaye, J., \& Pavel, M. (2009). Unobtrusive assessment of walking velocity in the home using inexpensive PIR sensors. Conf Proc IEEE Eng Med Biol Soc, (2009), 7248?7251. doi:10.1109/IEMBS.2009.5334746.

[2] Fritz, S., \& Lusardi, M. (2009). White paper: "Walking velocity: The Sixth Vital Sign". Journal of Geriatric Physical Therapy 32, 2 (2009), $2 ? 5$.

[3] Middleton, A., \& Lusardi, M. (2015). Walking velocity: The Functional Vital Sign. Journal of Aging and Physical Activity, (2015) 23(2): 314?322. doi:10.1123/japa.2013-0236.

[4] Kon, S., \& Jones, S. E., \& Schofield, S. J., \& Banya, W., \& Dickson, M. J., \& Canavan, J. L., \& Nolan, C. M., \& Haselden, B. M., \& Polkey, M. I., \& Cullinan, P, \& Man, W. D-C. (2015). Gait velocity and readmission following hospitalisation for acute exacerbations of COPD: a prospective study. Thorax 70, 12 (2015), 1131 ?1137.

[5] Schoon, Y., \& Bongers, J, \& Melis, R., \& Olde, R. M. (2014). Gait velocity as a test for monitoring frailty in community-dwelling older people has the highest diagnostic value compared to step length and chair rise time. European journal of physical and rehabilitation medicine 50, 6 (2014), 693?701.
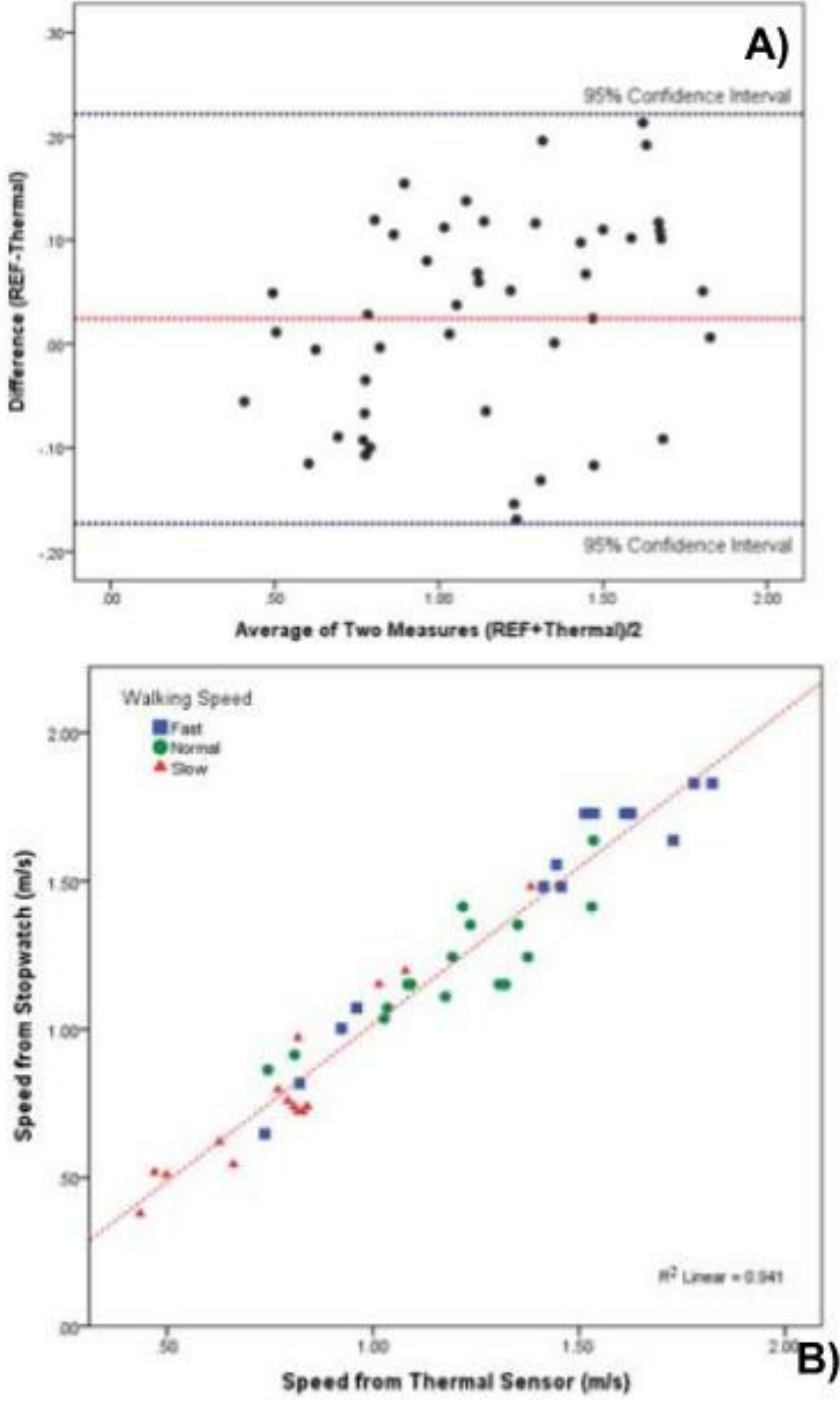

Fig. 5. Bland-Altman (A) and regression (B) plots illustrating the performance of the TVS for estimating walking velocity across a number of speeds. As illustrated in the plots, the model produces unbiased estimates of velocity when compared to the clinical standard of a human observer with stopwatch.

[6] Samah, Z. A., Nordin, N. A. M., Shahar, S., \& Singh, D. K. A. (2016) Can gait speed test be used as a falls risk screening tool in community dwelling older adults? A review. Polish Annals of Medicine, 23(1), 6167.

[7] Karpman, C., \& LeBrasseur, N. K., \& DePew, Z. S., \& Novotny, P. J., \& Benzo, R. P. (2013). Measuring gait velocity in the outpatient clinic: methodology and feasibility. Respiratory care, (2013), respcare?02688.

[8] Bethoux, F., \& Bennett, S. (2011).Evaluating Walking in Patients with Multiple Sclerosis. International Journal of MS Care, (2011) https://doi.org/10.7224/1537-2073-13.1.4 PMID: 24453700

[9] Steele, R., \& Lo, A., \& Secombe, C., \& Wong, Y. K. (2009). Elderly persons perception and acceptance of using wireless sensor networks to assist healthcare. International journal of medical informatics 78,12 (2009), 788 ?801.

[10] Muro-De-La-Herran, A., Garcia-Zapirain, B., \& Mendez-Zorrilla, A (2014). Gait analysis methods: An overview of wearable and nonwearable systems, highlighting clinical applications. Sensors, 14(2), 3362-3394.

[11] Gokalp, H., \& Clarke, M. (2013). Monitoring activities of daily living of the elderly and the potential for its use in telecare and telehealth: A review. TELEMEDICINE and e-HEALTH, 19, 12 (2013), 910 ?923. 
[12] Wing, M. G., \& Eklund, A., \& Kellogg, L. D. (2005). Consumer-grade global positioning system (GPS) accuracy and reliability. Journal of forestry 103, 4 (2005), 169?173.

[13] Heimann. (2017). Retrieved November 07, 2017, from http://www.heimannsensor.com/products_imaging.php

[14] Sixsmith, A., \& Johnson, N. (2004). A smart sensor to detect the falls of the elderly. IEEE Pervasive computing, 3(2), 42-47.

[15] Hsu, C., \& Liu, Y., \& Kebelac, Z., \& Hristov, R., \& Katabi, D., Liu, C. (2017). Extracting Gait Velocity and Stride Length from Surrounding Radio Signals. Human Factors in Computing Systems, 2116-2126, $10.1145 / 3025453.3025937$

[16] McGinnis, R.S., \& Mahadevan, N., \& Moon, Y., \& Seagers, K., \& Sheth, N., \& Wright, J. A. Jr., \& DiCristofaro, F. \& Silva, I., \& Jorthberg, E., Ceruolo, M., \& Pindado, J. A., \& Sosnoff, J., \& Ghaffari, R., \& Patel, S. (2017) A machine learning approach for gait velocity estimation using skin-mounted wearable sensors: From healthy controls to individuals with multiple sclerosis. PLoS ONE 12(6): e0178366. https://doi.org/10.1371/journal.pone.0178366

[17] Singh, O. I., \& Sinam, T. Local Contrast and Mean based Thresholding Technique in Image Binarization International Journal of Computer Applications.

[18] Suzuki, S. (1985). Topological structural analysis of digitized binary images by border following. Computer vision, graphics, and image processing, 30(1), 32-46.

[19] Ristic, B., Arulampalam, S., \& Gordon, N. J. (2004). Beyond the Kalman filter: Particle filters for tracking applications. Artech house.

[20] Bland, J. M., \& Altman, D. (1986). Statistical methods for assessing agreement between two methods of clinical measurement. The lancet, 327(8476), 307-310. 